\title{
Prediction Intervals for Progressive Type-II Right-Censored Order Statistics from Two Independent Sequences
}

\author{
M. M. Mohie El-Din ${ }^{1}$, M. S. Kotb ${ }^{1}$, W. S. Emam ${ }^{2}$ \\ ${ }^{1}$ Department of Mathematics, Faculty of Science, Al-Azhar, University, Nasr City, Cairo, Egypt \\ ${ }^{2}$ Department of Basic Science, Faculty of Engineering, British, University in Egypt, Al-Shorouq City, Cairo, Egypt
}

Email address:

mmmmoheeldin@yahoo.com (M. M. M. El-Din),kottp5_mas@yahoo.com (M. S. Kotb), walid_emam42@yahoo.com (W. S. Emam)

\section{To cite this article:}

M. M. Mohie El-Din, M. S. Kotb, W. S. Emam. Prediction Intervals for Progressive Type-II Right-Censored Order Statistics from Two Independent Sequences. American Journal of Theoretical and Applied Statistics. Vol. 4, No. 5, 2014, pp. 329-338.

doi: 10.11648/j.ajtas.20150405.13

\begin{abstract}
This article discusses the problem of predicting future progressive Type-II right censored order statistics based on progressive Type-II right-censored, ordered statistics, record values and current records that observed from the past X-sequence. Such coverage probabilities of the prediction intervals are exact and don't depend on the sampling distribution $F$. Finally, a real life time data were given to breakdown the insulating fluid between electrodes which is used to illustrate the derived results.
\end{abstract}

Keywords: Prediction Intervals, Progressive Censoring, Order Statistics, Records, Coverage Probability, Prediction Coefficient

\section{Introduction}

Statistical prediction is the derivation issue of the unknown values of future sample based on available observations. Prediction problems can be generally categorized as One-sample prediction, two-sample prediction and multi-sample prediction. Type II-sample prediction is governed here. In this type, predicting of future variables based on another independent observed sample. Also, the predictor can be either point or interval. The prediction (confidence) intervals can be one-sided or two-sided. Moreover, the predictor can be parametric (if it depends on the distribution parameters) or nonparametric (distribution-free prediction type). Distribution-free two-sided prediction intervals (DFPIs) are interest of natural in this article. Many contexts have taken place in the DFPIs direction in recent years by using several assumption; see, for example [1-11]. Recently, DFPIs for future progressively Type-II right-censored order statistics sample (PCOs) (which it's cdf purposed by Kamps and Cramer [12]) based on k-records [13]. Here, the DFPIs derivation for a future PCOs (that developed by Balakrishnan et al. [16]) based on different observed samples (PCOs; order statistics; upper records and largest current records).

This paper is organized as follows, section 2 contains some preliminaries. In section 3, the DFPIs discussion of a future
PCO based on PCOs, order statistics, upper records and current records. In section 4 , a real life time data are considered and numerical computations are given to illustrate most the results which are derived. Finally, the conclusion of this study is given in Section 5.

\section{Preliminaries}

Suppose $X_{1}, X_{2}, \ldots, X_{n}$ denote the observed lifetimes of identical units of size $n$ that placed on a reliability experiment of life-test. Assume that, these units are independent and identically distributed (iid) from a continuous population with cumulative distribution function (cdf) $F(x)$ and probability density function (pdf) $f(x)$. Further, independently of X-sample, let $Y_{1}, Y_{2}, \ldots, Y_{m}$ another unobserved sample of size $m$ that withdrawn from the same population, consider we intend to study $k$ units-out-of-m and remove from the life-test at different time points (progressively) the remaining $m-k$ units from these unobserved iid random variables (r.v's). Specifically, immediately following the first observed failure $Y_{1: k: m}^{*}$, remove from right at random $R_{1}$ surviving units from the test; next, immediately observe following the second failure $Y_{2: k: m}^{*}$, remove at random $R_{2}$ surviving units from the test, and so on; 
finally, after the $k^{\text {th }}$ observed failure $Y_{k: k: m}^{*}$, remove all the remaining surviving units (that did not failed yet) $R_{k}=n-k-\left(R_{k-1}+\ldots+R_{1}\right)$. Such $k$ the number of failures (that were already observed) and the progressive censoring scheme $\bar{R}=\left(R_{1}, \ldots, R_{k}\right)$ are pre-fixed, the scheme $Y_{1: k: m}^{*}, \ldots, Y_{k: k: m}^{*}$ is said to be progressive Type-II right-censoring, and the ordered values $Y_{1: k: m}<Y_{2: k: m}<\ldots<Y_{k: k: m} \quad$ were obtained from these observations are referred to PCOs. For more details concerning a PCOs, see, for example, Balakrishnan and Aggarwala [14], Balakrishnan [15]. The marginal pdf of the $r^{\text {th }} \mathrm{PCO}, Y_{r: k: m}(1 \leq r \leq k \leq m)$ that developed by Balakrishnan et al. [16], given by

$$
f_{Y_{r: k: m}}(y)=c_{r}^{\prime} \sum_{\ell=0}^{r-1} c_{\ell, r-1} \quad f(y)\{\bar{F}(y)\}^{R_{\ell, r^{\prime}}-1}
$$

where

$$
-\infty<y<\infty
$$

$c_{r}^{\prime}=m\left(m-R_{1}-1\right) \ldots\left(m-R_{1}-\ldots-R_{r-1}-r+1\right)$

$R_{\ell, r}^{\prime \prime}=\left(R_{r}^{*}+1\right)+\sum_{j=r-\ell}^{r-1}\left(R_{j}+1\right), \quad R_{r}^{*}=m-r-\left(R_{1}+\ldots+R_{r-1}\right)$, $c_{\ell, r}=c_{\ell, r}\left(R_{1}+1, \ldots, R_{r}+1\right)$

and

$\left(\prod_{j=1}^{\ell} \sum_{k=r-\ell+1}^{r-\ell+j}\left(R_{k}+1\right)\right)\left(\prod_{j=1}^{r-\ell} \sum_{k=j}^{r-\ell}\left(R_{k}+1\right)\right) c_{\ell, r}=(-1)^{\ell} \quad$. The survival function of $Y_{r: k: m}(1 \leq r \leq k \leq m)$ can be easily obtained as

$$
\bar{F}_{Y_{r: k: m}}(y)=c_{r}^{\prime} \sum_{\ell=0}^{r-1} \frac{c_{\ell, r-1}}{R_{\ell, r}^{\prime \prime}} \quad\{\bar{F}(y)\}^{R_{\ell, r}^{\prime}}
$$

It remains to refer that, not only the prediction coefficients $\varphi$ and $\pi$ which are stated here depend on the subscripts but also depend on observed sample size $n$, future PCOs size $k \leq m$ and the progressive censoring scheme $\bar{R}$, i.e.

$$
\varphi(.)=\varphi(., n ; k, m, \bar{R}), \quad \pi(.)=\pi(., n ; k, m, \bar{R}) .
$$

\section{DFPIs for Single PCO}

In this section, we intend to construct $100(1-\alpha) \%$ DFPIs for the $r^{\text {th }}$ PCO, $Y_{r: k: m}(1 \leq r \leq k \leq m)$ from the future PCOs of size $k$ out of $m$ from Y-sequence of the form $\left(X_{i}, X_{j}\right)$, such that, the lower $X_{i}$ and the upper $X_{j}$ bounded are observed from the following four schemes: PCOs, order statistics, upper records and current records, each case has separately discussion in special subsection, such that the corresponding coverage probabilities are free of the parent

\begin{tabular}{|c|c|c|c|c|c|c|c|c|c|c|}
\hline & & & \multicolumn{4}{|l|}{$\mathbf{j}$} & \multicolumn{4}{|l|}{$\mathbf{j}$} \\
\hline & & & 4 & 6 & 8 & 10 & 4 & 6 & 8 & 10 \\
\hline$m: k$ & $r$ & $i$ & \multicolumn{4}{|c|}{$(m-k, 0, \ldots, 0)$} & \multicolumn{4}{|c|}{$(0, \ldots, 0, m-k)$} \\
\hline \multirow[t]{6}{*}{$20: 10$} & 3 & 1 & 0.659 & 0.885 & 0.974 & 0.995 & 0.781 & 0.875 & 0.884 & 0.885 \\
\hline & & 2 & 0.444 & 0.670 & 0.733 & 0.741 & 0.424 & 0.517 & 0.527 & 0.527 \\
\hline & & 3 & 0.202 & 0.429 & 0.492 & 0.500 & 0.152 & 0.245 & 0.255 & 0.255 \\
\hline & 5 & 1 & 0.312 & 0.676 & 0.918 & 0.993 & 0.688 & 0.926 & 0.973 & 0.976 \\
\hline & & 2 & 0.265 & 0.629 & 0.872 & 0.946 & 0.494 & 0.731 & 0.778 & 0.782 \\
\hline & & 3 & 0.159 & 0.523 & 0.766 & 0.840 & 0.232 & 0.469 & 0.516 & 0.520 \\
\hline \multirow[t]{6}{*}{$20: 15$} & 9 & 1 & 0.116 & 0.435 & 0.811 & 0.985 & 0.284 & 0.698 & 0.945 & 0.996 \\
\hline & & 2 & 0.108 & 0.427 & 0.804 & 0.978 & 0.255 & 0.669 & 0.916 & 0.968 \\
\hline & & 3 & 0.077 & 0.396 & 0.772 & 0.946 & 0.164 & 0.577 & 0.825 & 0.877 \\
\hline & 11 & 1 & 0.031 & 0.198 & 0.581 & 0.940 & 0.139 & 0.501 & 0.864 & 0.993 \\
\hline & & 2 & 0.030 & 0.197 & 0.580 & 0.939 & 0.131 & 0.492 & 0.855 & 0.984 \\
\hline & & 3 & 0.023 & 0.191 & 0.574 & 0.932 & 0.092 & 0.454 & 0.817 & 0.946 \\
\hline \multirow[t]{6}{*}{$15: 8$} & 4 & 1 & 0.345 & 0.691 & 0.916 & 0.989 & 0.655 & 0.906 & 0.968 & 0.974 \\
\hline & & 2 & 0.284 & 0.629 & 0.824 & 0.928 & 0.469 & 0.720 & 0.781 & 0.788 \\
\hline & & 3 & 0.163 & 0.509 & 0.734 & 0.807 & 0.223 & 0.474 & 0.536 & 0.542 \\
\hline & 6 & 1 & 0.070 & 0.277 & 0.624 & 0.934 & 0.398 & 0.780 & 0.962 & 0.996 \\
\hline & & 2 & 0.065 & 0.272 & 0.619 & 0.929 & 0.336 & 0.718 & 0.900 & 0.934 \\
\hline & & 3 & 0.045 & 0.252 & 0.600 & 0.910 & 0.197 & 0.579 & 0.760 & 0.794 \\
\hline \multirow[t]{6}{*}{$25: 15$} & 4 & 1 & 0.690 & 0.913 & 0.962 & 0.966 & 0.800 & 0.905 & 0.915 & 0.915 \\
\hline & & 2 & 0.477 & 0.700 & 0.748 & 0.753 & 0.459 & 0.563 & 0.573 & 0.574 \\
\hline & & 3 & 0.218 & 0.441 & 0.489 & 0.494 & 0.169 & 0.274 & 0.284 & 0.284 \\
\hline & 6 & 1 & 0.432 & 0.804 & 0.967 & 0.995 & 0.710 & 0.937 & 0.976 & 0.978 \\
\hline & & 2 & 0.359 & 0.732 & 0.895 & 0.923 & 0.510 & 0.737 & 0.776 & 0.778 \\
\hline & & 3 & 0.206 & 0.578 & 0.741 & 0.769 & 0.237 & 0.464 & 0.503 & 0.505 \\
\hline
\end{tabular}
distribution $F$.

\subsection{Based on PCOs}

Table 1. values of $\pi_{1}(i, j, \mu ; r)$ for $n: \mu=20: 10$ and $\bar{R}_{1}^{*}=(10,0, \ldots, 0)$ for some choices of $m, k, \bar{R}, i, j$ and $r$ 
Let $X_{1: \mu: n}<X_{2: \mu: n}<\ldots<X_{\mu: \mu: n}$ be an observed sequence of PCOs of size $\mu$ from the $X$-sequence with the progressive censoring scheme $\bar{R}^{*}=\left(R_{1}^{*}, \ldots, R_{\mu}^{*}\right)$. Suppose we are interested in obtaining $100(1-\alpha) \%$ DFPIs for $Y_{r: k: m}$ of the form $\left(X_{i: \mu: n}, X_{j: \mu: n}\right)$, such that the coverage probability $p\left(X_{i: \mu: n} \leq Y_{r: k: m} \leq X_{j: \mu: n}\right)=1-\alpha$ being free of the parent distribution $F$.

Lemma 1. Let $\quad\left\{X_{i: \mu: n}, 1 \leq i \leq \mu \leq n\right\} \quad$ and $\left\{Y_{j: k: m}, 1 \leq j \leq k \leq m\right\}$ be two independent PCOs from

$$
\varphi_{1}\left(i, \mu, \bar{R}^{*} ; r\right)=p\left(X_{i: \mu: n} \geq Y_{r: k: m}\right)=\int_{-\infty}^{\infty} p\left(X_{i: \mu: n} \geq y\right) f_{Y_{r: k: m}}(y) d y=c_{r}^{\prime} c_{i}^{*} \sum_{v=0}^{r-1} \sum_{\ell=0}^{i-1} \frac{c_{v, r-1} c_{\ell, i-1}^{*}}{R_{\ell, i}^{* \prime \prime}} \int_{0}^{1} y^{R_{\ell, i}^{* \prime}+R_{v, r}^{\prime \prime}-1} d y .
$$
one-sided PI for the future $Y_{r: k: m}$, with the corresponding sampling distribution $F$, and is given by

$$
\varphi_{1}\left(i, \mu, \bar{R}^{*} ; r\right)=c_{r}^{\prime} c_{i}^{*^{\prime}} \sum_{v=0}^{r-1} \sum_{\ell=0}^{i-1} \frac{c_{v, r-1} c_{\ell, i-1}^{*}}{R_{\ell, i}^{*^{\prime \prime}}\left(R_{\ell, i}^{*^{\prime \prime}}+R_{v, r}^{\prime^{\prime}}\right)} .
$$

Proof: Upon (1) and (2), we can write continuous cdf $F$. then $\left(-\infty, X_{i: \mu: n}\right), 1 \leq i \leq \mu \leq n$, is DF

\begin{tabular}{|c|c|c|c|c|c|c|c|c|c|c|}
\hline & & & \multicolumn{4}{|l|}{$\mathbf{j}$} & \multicolumn{4}{|l|}{$\mathbf{j}$} \\
\hline & & & $\mathbf{r + 3}$ & $r+4$ & $\mathbf{r + 5}$ & $r+6$ & $\mathbf{r + 3}$ & $\mathbf{r + 4}$ & $\mathbf{r + 5}$ & $r+6$ \\
\hline$m: k$ & $r$ & $i$ & \multicolumn{4}{|c|}{$(0,0,2,3,0, \ldots, 0)$} & \multicolumn{4}{|c|}{$(0, \ldots, 0,5)$} \\
\hline \multirow[t]{9}{*}{$15: 10$} & 4 & $r-3$ & 0.472 & 0.584 & 0.684 & 0.768 & 0.504 & 0.616 & 0.714 & 0.794 \\
\hline & & $\mathrm{r}-2$ & 0.449 & 0.561 & 0.660 & 0.744 & 0.477 & 0.589 & 0.687 & 0.768 \\
\hline & & $\mathrm{r}-1$ & 0.405 & 0.417 & 0.617 & 0.701 & 0.428 & 0.541 & 0.638 & 0.719 \\
\hline & 6 & $\mathrm{r}-3$ & 0.199 & 0.273 & 0.356 & 0.444 & 0.372 & 0.477 & 0.579 & 0.674 \\
\hline & & $\mathrm{r}-2$ & 0.192 & 0.267 & 0.350 & 0.438 & 0.355 & 0.459 & 0.562 & 0.657 \\
\hline & & $\mathrm{r}-1$ & 0.177 & 0.252 & 0.335 & 0.423 & 0.319 & 0.423 & 0.526 & 0.621 \\
\hline & 8 & $\mathrm{r}-3$ & 0.057 & 0.088 & 0.127 & 0.179 & 0.267 & 0.358 & 0.457 & 0.557 \\
\hline & & $\mathrm{r}-2$ & 0.056 & 0.086 & 0.126 & 0.177 & 0.254 & 0.345 & 0.444 & 0.544 \\
\hline & & $\mathrm{r}-1$ & 0.052 & 0.082 & 0.123 & 0.174 & 0.231 & 0.322 & 0.421 & 0.521 \\
\hline \multirow[t]{9}{*}{$25: 20$} & 10 & $r-3$ & 0.525 & 0.627 & 0.716 & 0.789 & 0.636 & 0.713 & 0.770 & 0.808 \\
\hline & & $\mathrm{r}-2$ & 0.475 & 0.577 & 0.666 & 0.739 & 0.549 & 0.625 & 0.682 & 0.721 \\
\hline & & $\mathrm{r}-1$ & 0.406 & 0.508 & 0.597 & 0.670 & 0.442 & 0.519 & 0.576 & 0.615 \\
\hline & 14 & $\mathrm{r}-3$ & 0.430 & 0.545 & 0.656 & 0.755 & 0.619 & 0.697 & 0.754 & 0.792 \\
\hline & & $\mathrm{r}-2$ & 0.398 & 0.513 & 0.624 & 0.723 & 0.535 & 0.613 & 0.670 & 0.708 \\
\hline & & $\mathrm{r}-1$ & 0.352 & 0.466 & 0.577 & 0.677 & 0.434 & 0.512 & 0.569 & 0.607 \\
\hline & 18 & $\mathrm{r}-3$ & 0.305 & 0.442 & 0.603 & 0.769 & 0.659 & 0.740 & 0.792 & 0.820 \\
\hline & & $\mathrm{r}-2$ & 0.293 & 0.430 & 0.591 & 0.757 & 0.576 & 0.656 & 0.708 & 0.736 \\
\hline & & $\mathrm{r}-1$ & 0.272 & 0.409 & 0.570 & 0.736 & 0.472 & 0.552 & 0.604 & 0.632 \\
\hline
\end{tabular}
prediction coefficient $\varphi_{1}(i, \mu ; r)$, that does not depend on the

Table 2. values of $\pi_{1}(i, j, \mu ; r)$ for $n: \mu=30: 25$ and $\bar{R}^{*}=(0,0,2,3,0, \ldots, 0)$ for some choices of $m, k, \bar{R}, i, j$ and $r$.

Theorem 1. Under the assumption of lemma 1, then $\left(X_{i: \mu: n}, X_{j: \mu: n}\right) \quad, \quad 1 \leq i<j \leq \mu \leq n \quad$ is a DFPIs for $Y_{r: k: m}(1 \leq j \leq k \leq m)$, whose coverage probability is free of the 


\subsection{Based on Order Statistics}

In the following, the prediction discussion of future PCOs based on observed order statistics. Let $X_{1}, X_{2}, \ldots, X_{n}$ denote sequences of the lifetimes of reliability experiment units, we shall assume that these variables are iid from an absolutely continuous population with cdf $F$. Suppose $X_{1: n} \leq X_{2: n} \leq \ldots \leq X_{n: n}$ are usual order statistics that obtained from these iidr.v's, for more details about order statistics, see, Arnold et al. [17]. The marginal pdf and the survival function of $X_{i: n}$ are easily expressed in terms of $F$ and $f$, respectively as.

$$
\begin{aligned}
& f_{X_{i: n}}(x)=i\left(\begin{array}{l}
n \\
i
\end{array}\right)[F(x)]^{i-1}[\bar{F}(x)]^{n-i} f(x), \quad \text { (6) } \begin{array}{r}
\text { Upon the conditioning argument, in the } \\
\text { prediction procedure by assuming that } \\
\text { continuous r.v's also, we then have }
\end{array} \\
& \bar{F}_{X_{i: n}}(x)=\sum_{\ell=0}^{i-1}\left(\begin{array}{l}
n \\
i
\end{array}\right)[F(y)]^{\ell}[\bar{F}(y)]^{n-\ell} . \quad \text { (7) } \\
& \pi(i, j ; r)=P\left(X_{i} \leq Y_{r} \leq X_{j}\right)=\int_{-\infty}^{\infty} P\left(X_{i} \leq Y_{r} \leq X_{j} \mid Y_{r}=y\right) d F_{Y_{r}}(y)=\int_{-\infty}^{\infty} p\left(X_{i} \leq y \leq X_{j}\right) f_{Y_{r}}(y) d y .
\end{aligned}
$$

Lemma 2.

Based on X-sample observations, suppose we are interested in obtaining $(1-\alpha) 100 \%$ DFPIs for $Y_{r}$ from a future Y-sample of the form $\left(X_{i}, X_{j}\right), 1 \leq i<j$, such that, the coverage probability $P\left(X_{i} \leq Y_{r} \leq X_{j}\right)=1-\alpha$. We refer to the interval $\left(X_{i}, X_{j}\right)$ as $(1-\alpha) 100 \%$ PI for $Y_{r}$. For a given $y$, if $X_{i}$ and $X_{j}$ are continuous r.v's, we get

$$
\begin{aligned}
p\left(X_{i} \leq y \leq X_{j}\right) & =P\left(X_{j} \geq y\right)-P\left(X_{i} \geq y\right) \\
& =\bar{F}_{X_{j}}(y)-\bar{F}_{X_{i}}(y)
\end{aligned}
$$

Upon the conditioning argument, in the non-parametric prediction procedure by assuming that $X_{i}$ and $X_{j}$ are
Such that, $\pi(i, j ; r)=1-\alpha$ represent the prediction coefficient which does not depend on the parameters of the parent distribution $F$, wich it's depends only on the r.v's positions (the indices $i, j$ and $r$ ). Here, $X_{i}$ and $X_{j}$ are the lower and upper bounds of the prediction interval for $Y_{r}$ respectively.

Theorem 2. Let $X_{i: n}(1 \leq i \leq n)$ be $i^{\text {th }}$ order statistic from an observed random sample of size $n$ with continuous $\operatorname{cdf} F$. If $Y_{r: k: m}, 1 \leq r \leq k \leq m$ is the $r$ th PCOs from a future unobserved random sample of size $k$-out-of $m$ with the same $\operatorname{cdf} F$, then $\left(X_{i: n}, X_{j: n}\right), 1 \leq i<j \leq n$, is a DFPI for $Y_{r: k: m}$, whose coverage probability is free of $F$ and the corresponding prediction coefficient $\pi_{2}$ is given by

$$
\pi_{2}(i, j ; r)=c_{r}^{\prime} \sum_{\ell=i}^{j-1} \sum_{v=0}^{r-1}\left(\begin{array}{l}
n \\
\ell
\end{array}\right) c_{v, r-1}\left\{\left(n-\ell+R_{v, r}^{\prime \prime}\right)\left(\begin{array}{l}
n+R_{v, r}^{\prime \prime} \\
\ell
\end{array}\right)\right\}^{-1}
$$

Proof: Such $X_{i: n}$ is continuous, it is known from (7) and (8) that

$$
P\left(X_{i: n} \leq y \leq X_{j: n}\right)=\sum_{\ell=i}^{j-1}\left(\begin{array}{l}
n \\
\ell
\end{array}\right)(F(y))^{\ell}(1-F(y))^{n-\ell} .
$$

By conditioning on $Y_{r: k: m}=y$, and from (6) and (9), it follows that

$$
P\left(X_{i: n} \leq Y_{r: k: m} \leq X_{j: n}\right)=c_{r}{ }^{\prime} \sum_{\ell=i}^{j-1} \sum_{v=0}^{r-1}\left(\begin{array}{l}
n \\
\ell
\end{array}\right) c_{v, r-1} \int_{-\infty}^{\infty}(F(y))^{\ell}(1-F(y))^{n+R_{r, v}^{\prime \prime}-\ell-1} f(y) d y=c_{r}{ }^{\prime} \sum_{\ell=i}^{j-1} \sum_{\nu=0}^{r-1}\left(\begin{array}{l}
n \\
\ell
\end{array}\right) c_{v, r-1} B\left(\ell+1, n-\ell+R_{v, r}^{\prime \prime}\right) .
$$

Equation (10) can be obtained directly by simplifying the previous beta constants, we have presented the values of $\pi_{2}(i, j ; r)$ that given by (10) for $m: k=20: 10$ and for some selected values of the integers $n, i, j, \bar{R}$ and $r$ in table 3. Thus, $\left(X_{i: n}, X_{j: n}\right)$ is a $\left(\pi_{2}\right) 100 \%$ DFPI for the future $r^{\text {th }}$ PCOs from a future unobserved random sample of size $k$, with $\pi_{2}(i, j ; r)$ given by (10) which does not depend on $F$.

\subsection{Based on Upper Records}

Here, The prediction discussion of future PCOs based on observed upper records. Let $X_{i},(i \geq 1)$ be a sequence of iid continuous r.v's with cdf $F$. An observation $X_{j}$ is defined to be an upper record if $X_{j}>X_{i}$ for every $i<j$, one may refer to the books by Arnold et al.[17], Nevzorov[18], Gulati and Padgett[19] and Ahsanullah[20]. Let us denote the $j^{\text {th }}$ upper record value by $U_{j}$. Then the survival function of of the $U_{j}$ is given by

$$
\bar{F}_{U_{j}}(u)=\bar{F}(u) \sum_{\ell=0}^{j-1} \frac{\{-\log \bar{F}(u)\}^{\ell}}{\ell !} .
$$


Table 3. The values of $\pi_{2}(i, j ; r)$ for $m: k=20: 10$ and for some selected choices of $n, i, j, \bar{R}$ and $r$.

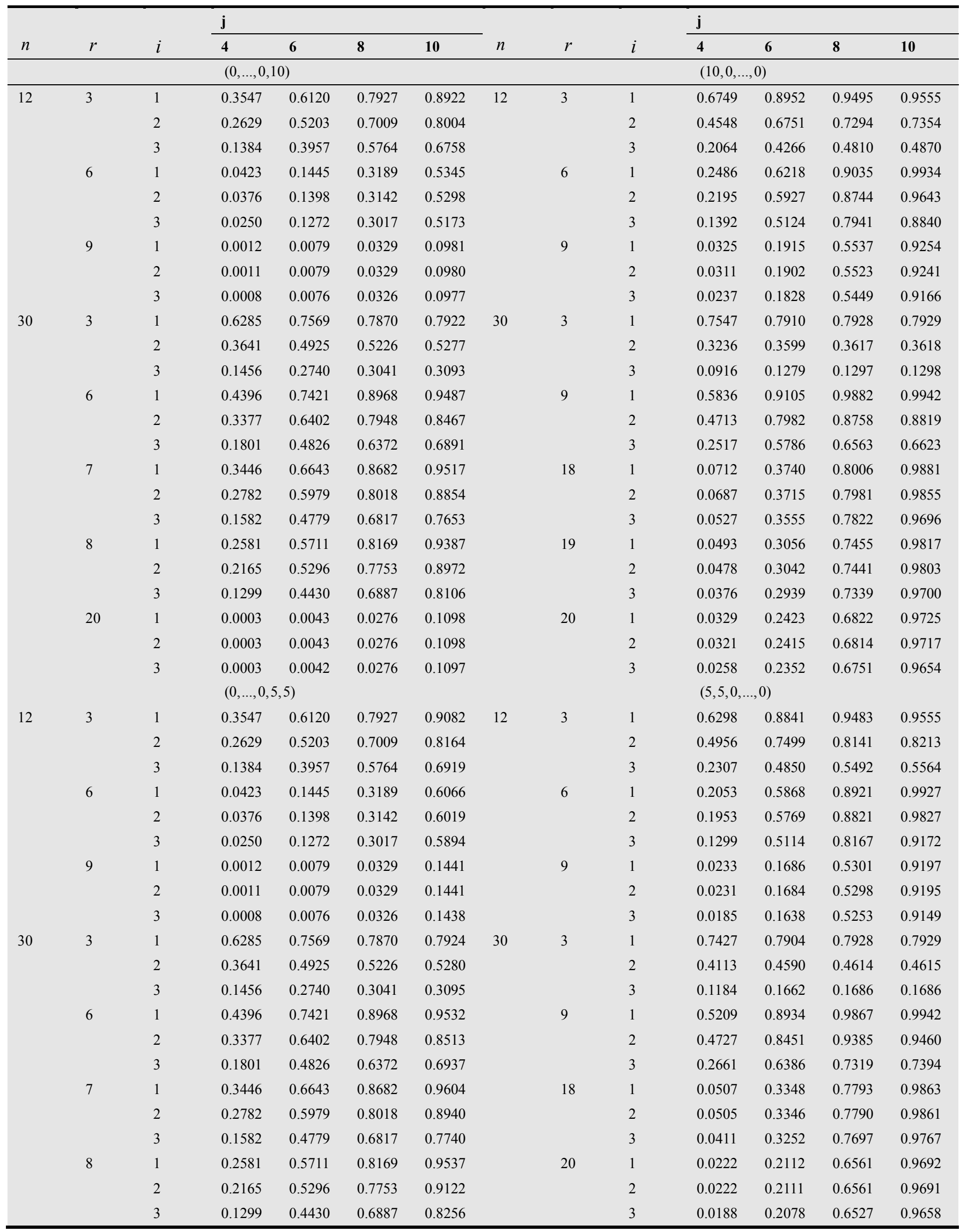


Table 4. The values of $\pi_{2}(i, j ; r)$ for $m: k=20: 10$ and for some selected choices of $n, i, j, \bar{R}$ and $r$.

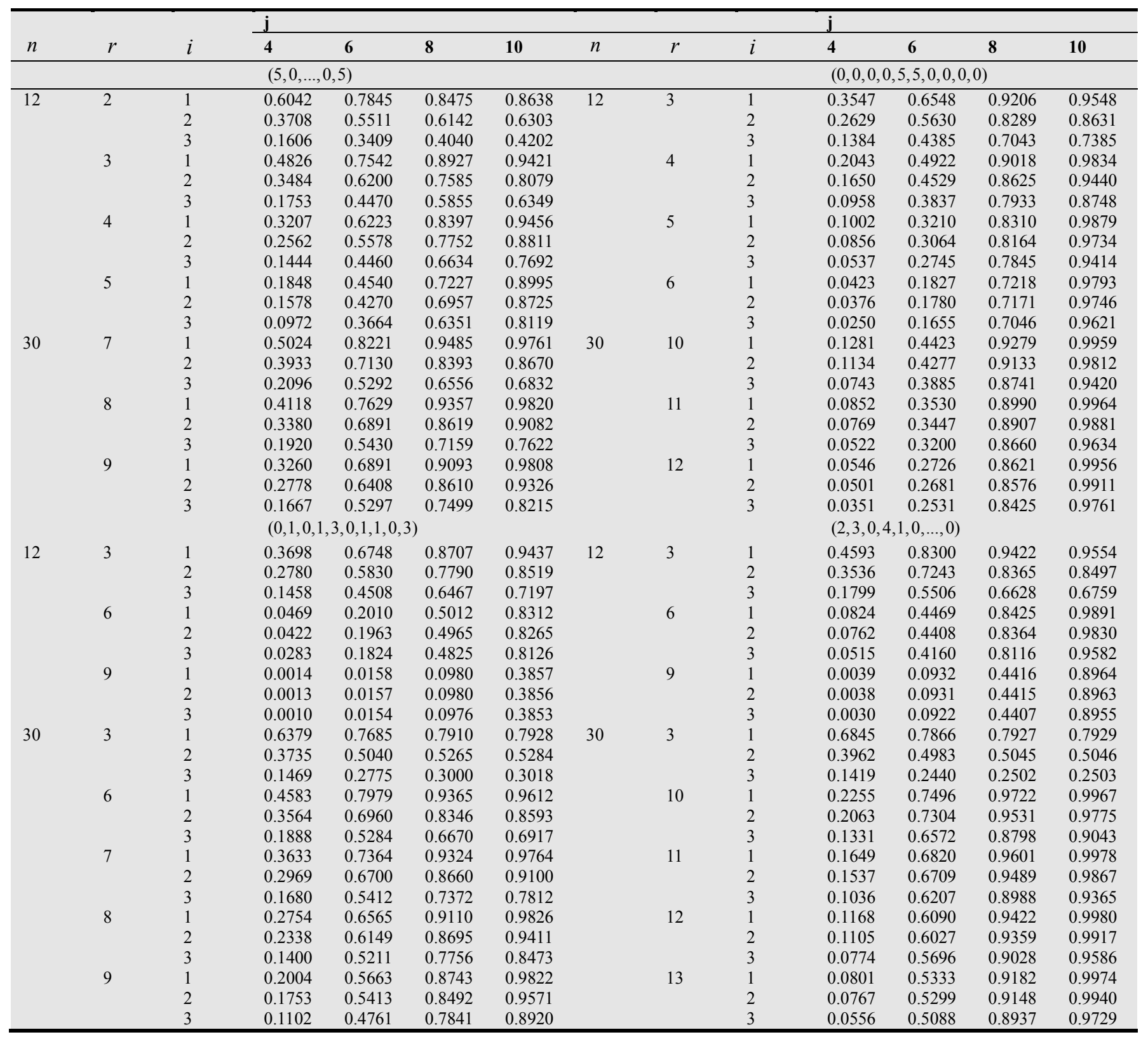

Theorem 3. Let $U_{j},(j \geq 1)$ be $j^{\text {th }}$ upper record from an observed random sample of size $n$ with continuous $\operatorname{cdf} F$. If $Y_{r: k: m}, 1 \leq r \leq k \leq m$ is the $r$ th PCOs from a future unobserved sample of size $k$ with the same $\operatorname{cdf} F$, then $\left(U_{i}, U_{j}\right), 1 \leq i<j$, is DFPI for the future $r^{\text {th }}$ PCOs, $X_{r: k: m}$, whose coverage probability is free from $F$, and is given by

$$
\begin{gathered}
\pi_{3}(i, j ; r)=c_{r} \cdot \sum_{\ell=i}^{j-1} \sum_{\nu=0}^{r-1} \frac{c_{v, r-1}}{\left(R_{v, r}^{\prime \prime}+1\right)^{\ell+1}} . \quad \text { (13) } \quad \text { and (9), we readily obtain } \\
p\left(U_{i} \leq Y_{r: k: m} \leq U_{j}\right)=\int_{0}^{1} y \sum_{\ell=i}^{j-1} \frac{\{-\log y\}^{\ell}}{\ell !} c_{r}^{\prime} \sum_{\nu=0}^{r-1} c_{v, r-1} y^{R_{v, r}^{\prime}-1} d y=c_{r}^{\prime} \sum_{\ell=i}^{j-1} \sum_{\nu=0}^{r-1} c_{v, r-1} \int_{0}^{1} \frac{\{-\log y\}^{\ell}}{\ell !} y^{R_{v, r}^{\prime \prime}} d y .
\end{gathered}
$$

Proof: For a given $\mathrm{y}$, by assuming that the records $U_{j},(j \geq 1)$ are continuous r.v's, and from (12) and (8) it is now easy to write (see,[6])

$$
p\left(U_{i} \leq y \leq U_{j}\right)=\bar{F}(y) \sum_{\ell=i}^{j-1} \frac{\{-\log \bar{F}(y)\}^{\ell}}{\ell !} .
$$

The same method as a proof of Theorem 1; and from (14)

The values of $\pi_{3}(i, j ; r)$ are presented in table 5 for $m: k=30: 25 ; 50: 45$ and for some selected choices of 
$i, j, \bar{R}$ and $r .\left(U_{i}, U_{j}\right)$ is a $\left(\pi_{3}\right) 100 \%$ PIs for the future $r^{\text {th }}$ PCOs, such $\pi_{3}(i, j ; r)$ given by (13).

\subsection{Based on Current Records}

Suppose $X_{i},(i \geq 1)$ is a sequence of iid continuous r.v's with $\operatorname{cdf} F$. Let us denote the $i^{\text {th }}$ upper records by $U_{i}$ (with $U_{1} \equiv R_{0}^{\ell} \equiv X_{1}$ ). Now, let $R_{m}^{\ell}$ be the largest observation, at the time when the $i^{\text {th }}$ upper record occurs in the $X$-sequence. Then, the marginal density and the survival functions of $R_{i}^{\ell}$ (see Arnold et al. [21], p. 276), are given respectively by

$$
\begin{gathered}
f_{R_{i}^{\ell}}(y)=2^{i} f(y)\left(1-\bar{F}(y) \sum_{\lambda=0}^{i-1} \frac{\{-\log \bar{F}(y)\}^{\lambda}}{\lambda !}\right), \\
\bar{F}_{R_{i}^{\ell}}(u)=\bar{F}(u)\left[2^{i}-\bar{F}(u) \sum_{\lambda=0}^{i-1}\left(2^{i}-2^{\lambda}\right) \frac{\{-\log \bar{F}(u)\}^{\lambda}}{\lambda !}\right] .
\end{gathered}
$$

DFPI of future PCOs based on observed upper records are discussed as the same method of Theorem 1 In the following, let $Y_{r: k: m}$ be a future $r^{\text {th }}$ PCOs from the Y-sequence that we are interested in obtaining $100(1-\alpha) \%$ PIs for it of the form $\left(R_{i}^{l}, R_{j}^{l}\right)$ such that $R_{j}^{l}$ is the $j^{\text {th }}$ largest record and $p\left(R_{i}^{l} \leq y \leq R_{j}^{l}\right) \geq 1-\alpha$.

Lemma 3.

Let $R_{j}^{l},(j \geq 1)$ be $j^{\text {th }}$ largest current record from an observed random sample with continues $\operatorname{cdf} F$. If $Y_{r: k: m}$, $1 \leq r \leq k \leq m$ is the $r$ th PCOs from a future unobserved random sample of size $k$ with the same $\operatorname{cdf} F$, then $\left(-\infty, R_{i}^{l}\right), 1 \leq i \leq j$, is DF one-sided PI for the future $Y_{r: k: m}$, with the corresponding prediction coefficient $\varphi_{2}(i ; r)$, that does not depend on $F$, and is given by

$$
\varphi_{2}(i ; r)=c_{r}^{\prime} \sum_{v=0}^{r-1} c_{v, r-1}\left[\frac{2^{i}}{R_{v}^{\prime \prime}+1}-\sum_{\lambda=0}^{i-1} \frac{\left(2^{i}-2^{\lambda}\right)}{\left\{R_{v}^{\prime \prime}+2\right\}^{\lambda+1}}\right] .
$$

Proof: From (17) and (1), we get

$$
\varphi_{2}(i ; r)=p\left(R_{i}^{l} \geq Y_{r: k: m}\right)=\int_{-\infty}^{\infty} p\left(R_{i}^{l} \geq y\right) f_{Y_{r: k: m}}(y) d y=c_{r}^{r} \sum_{v=0}^{r-1} c_{v, r-1}\left[2^{i} \int_{0}^{1} y^{R_{v}^{\prime}} d y-\sum_{\lambda=0}^{i-1}\left(2^{i}-2^{\lambda}\right) \int_{0}^{1} y^{R_{v}^{\prime}+1} \frac{\{-\log y\}^{\lambda}}{\lambda !} d y\right]
$$

\begin{tabular}{|c|c|c|c|c|c|c|c|c|c|c|c|}
\hline \multirow[b]{2}{*}{$m: k$} & \multirow[b]{2}{*}{$r$} & \multirow[b]{2}{*}{$i$} & \multicolumn{4}{|l|}{$\mathbf{j}$} & \multirow[b]{2}{*}{$r$} & \multirow[b]{2}{*}{$i$} & \multicolumn{3}{|l|}{$\mathbf{j}$} \\
\hline & & & 15 & 25 & 35 & $m: k$ & & & 15 & 25 & 35 \\
\hline & & & \multicolumn{4}{|c|}{$(2,2,1,0, \ldots, 0)$} & \multicolumn{5}{|c|}{$(5,0, \ldots, 0)$} \\
\hline \multirow[t]{12}{*}{$: 25$} & 10 & 1 & 0.3700 & 0.3700 & 0.3700 & $50: 45$ & 30 & 1 & 0.6514 & 0.6514 & 0.6514 \\
\hline & & 2 & 0.0858 & 0.0858 & 0.0858 & & & 2 & 0.2910 & 0.2910 & 0.2910 \\
\hline & & 3 & 0.0150 & 0.0150 & 0.0150 & & & 3 & 0.0980 & 0.0980 & 0.0980 \\
\hline & 15 & 1 & 0.5669 & 0.5669 & 0.5669 & & 40 & 1 & 0.8693 & 0.8693 & 0.8693 \\
\hline & & 2 & 0.2152 & 0.2152 & 0.2152 & & & 2 & 0.6125 & 0.6125 & 0.6125 \\
\hline & & 3 & 0.0622 & 0.0622 & 0.0622 & & & 3 & 0.3516 & 0.3516 & 0.3516 \\
\hline & 20 & 1 & 0.7938 & 0.7938 & 0.7938 & & 43 & 1 & 0.9346 & 0.9346 & 0.9346 \\
\hline & & 2 & 0.4373 & 0.4373 & 0.4373 & & & 2 & 0.7659 & 0.7659 & 0.7659 \\
\hline & & 3 & 0.1982 & 0.1982 & 0.1982 & & & 3 & 0.5396 & 0.5396 & 0.5396 \\
\hline & 25 & 1 & 0.9599 & 0.9599 & 0.9599 & & 45 & 1 & 0.9769 & 0.9769 & 0.9769 \\
\hline & & 2 & 0.8484 & 0.8491 & 0.8484 & & & 2 & 0.9025 & 0.9038 & 0.9025 \\
\hline & & 3 & 0.7606 & 0.7606 & 0.7606 & & & 3 & 0.7687 & 0.7687 & 0.7700 \\
\hline
\end{tabular}

Table 5. The values of $\pi_{3}(i, j ; r)$ for $m: k=30: 25 ; 50: 45$ and for some selected choices of $i, j, \bar{R}$ and $r$

Theorem 4. Under the assumption of lemma 3, then $\left(R_{i}^{l}, R_{j}^{l}\right), 1 \leq i \leq j$, is DFPI for the future $Y_{r: k: m}$, with the corresponding prediction coefficient $\pi_{4}(i, j ; r)$ does not

$$
\pi_{4}(i, j ; r)=p\left(R_{i}^{l} \leq Y_{r: k: m} \leq R_{j}^{l}\right)=p\left(R_{j}^{l} \geq Y_{r: k: m}\right)-p\left(R_{i}^{l} \geq Y_{r: k: m}\right)=\varphi_{2}(j ; r)-\varphi_{2}(i ; r) .
$$


$i, j, \bar{R}$ and $r$. Such $\pi_{4}(i, j ; r)$ is given by (20) which does not depend on the parent distribution $F$.

Table 6. The values of $\pi_{4}(i, j ; r)$ for $m: k=30: 25 ; 50: 45$ and for some selected choices of $i, j, \bar{R}$ and $r$.

\begin{tabular}{|c|c|c|c|c|c|c|c|c|c|c|}
\hline \multirow{3}{*}{$m: k$} & \multirow[b]{3}{*}{$r$} & & \multicolumn{4}{|l|}{$\mathbf{j}$} & \multicolumn{4}{|l|}{$\overline{\mathbf{j}}$} \\
\hline & & & 15 & 25 & 35 & 45 & 15 & 25 & 35 & 45 \\
\hline & & $i$ & \multicolumn{4}{|c|}{$(0,2,0,3,0, \ldots, 0)$} & \multicolumn{4}{|c|}{$(5,0, \ldots, 0)$} \\
\hline \multirow[t]{12}{*}{$30: 25$} & \multirow[t]{3}{*}{10} & 1 & 0.145 & 0.145 & 0.145 & 0.145 & 0.154 & 0.154 & 0.154 & 0.154 \\
\hline & & 2 & 0.046 & 0.046 & 0.046 & 0.046 & 0.050 & 0.810 & 0.870 & 0.932 \\
\hline & & 3 & 0.012 & 0.012 & 0.012 & 0.012 & 0.014 & 0.014 & 0.014 & 0.014 \\
\hline & \multirow[t]{3}{*}{15} & 1 & 0.331 & 0.331 & 0.331 & 0.331 & 0.339 & 0.339 & 0.339 & 0.339 \\
\hline & & 2 & 0.160 & 0.160 & 0.160 & 0.160 & 0.166 & 0.166 & 0.166 & 0.166 \\
\hline & & 3 & 0.066 & 0.066 & 0.066 & 0.066 & 0.070 & 0.070 & 0.070 & 0.070 \\
\hline & \multirow[t]{3}{*}{20} & 1 & 0.590 & 0.590 & 0.590 & 0.590 & 0.596 & 0.596 & 0.596 & 0.596 \\
\hline & & 2 & 0.403 & 0.403 & 0.403 & 0.403 & 0.409 & 0.409 & 0.409 & 0.409 \\
\hline & & 3 & 0.244 & 0.244 & 0.244 & 0.244 & 0.250 & 0.250 & 0.250 & 0.250 \\
\hline & \multirow[t]{3}{*}{25} & 1 & 0.899 & 0.924 & 0.924 & 0.924 & 0.900 & 0.925 & 0.925 & 0.925 \\
\hline & & 2 & 0.840 & 0.865 & 0.866 & 0.866 & 0.842 & 0.867 & 0.868 & 0.868 \\
\hline & & 3 & 0.759 & 0.783 & 0.784 & 0.784 & 0.761 & 0.786 & 0.787 & 0.787 \\
\hline \multirow[t]{12}{*}{$50: 45$} & \multirow[t]{3}{*}{30} & 1 & 0.427 & 0.427 & 0.427 & 0.427 & 0.429 & 0.429 & 0.429 & 0.429 \\
\hline & & 2 & 0.234 & 0.234 & 0.234 & 0.234 & 0.236 & 0.236 & 0.236 & 0.236 \\
\hline & & 3 & 0.110 & 0.110 & 0.110 & 0.110 & 0.111 & 0.111 & 0.111 & 0.111 \\
\hline & \multirow[t]{3}{*}{35} & 1 & 0.580 & 0.580 & 0.580 & 0.580 & 0.582 & 0.582 & 0.582 & 0.582 \\
\hline & & 2 & 0.387 & 0.387 & 0.387 & 0.387 & 0.389 & 0.389 & 0.389 & 0.389 \\
\hline & & 3 & 0.226 & 0.226 & 0.226 & 0.226 & 0.227 & 0.227 & 0.227 & 0.227 \\
\hline & \multirow[t]{3}{*}{40} & 1 & 0.757 & 0.757 & 0.757 & 0.757 & 0.758 & 0.758 & 0.758 & 0.758 \\
\hline & & 2 & 0.606 & 0.606 & 0.606 & 0.606 & 0.608 & 0.608 & 0.608 & 0.608 \\
\hline & & 3 & 0.442 & 0.442 & 0.442 & 0.442 & 0.443 & 0.443 & 0.443 & 0.443 \\
\hline & \multirow[t]{3}{*}{45} & 1 & 0.914 & 0.956 & 0.957 & 0.957 & 0.914 & 0.956 & 0.957 & 0.957 \\
\hline & & 2 & 0.877 & 0.920 & 0.921 & 0.921 & 0.877 & 0.920 & 0.921 & 0.921 \\
\hline & & 3 & 0.821 & 0.864 & 0.865 & 0.865 & 0.821 & 0.864 & 0.865 & 0.865 \\
\hline
\end{tabular}

\section{Real Life Data Example}

The real life-observations that given in Nelson [22] are considered in this section to illustrate the derived results. These data which was also used in Lawless ([23], p. 185), The observation is data time to breakdown of an insulating fluid between electrodes at a voltage of $34 \mathrm{kV}$ (minutes). The 19 times to breakdown are contained in the following table $\left(^{*}\right)$

\begin{tabular}{llllllllll}
\hline 0.96 & 4.15 & 0.19 & 0.78 & 8.01 & 31.75 & 7.35 & 6.50 & 8.27 & 33.91 \\
32.52 & 3.16 & 4.85 & 2.78 & 4.67 & 1.31 & 12.06 & 36.71 & 72.89 & \\
\hline
\end{tabular}

The observed PCOs from the data $\left(^{*}\right)$ as $n: \mu=19: 14$ with the progressive censoring scheme $(0, \ldots, 0,5)$ can be:

\begin{tabular}{lllllll}
\hline 0.19 & 0.78 & 0.96 & 2.78 & 3.16 & 4.15 & 4.85 \\
6.50 & 7.35 & 8.01 & 8.27 & 31.75 & 32.52 & 33.91 \\
\hline
\end{tabular}

Also, the usual order statistics values that obtained from the sample $(*)$ will be:

\begin{tabular}{|c|c|c|c|c|c|c|c|c|c|}
\hline 0.19 & 0.78 & 0.96 & 1.31 & 2.78 & 3.16 & 4.15 & 4.67 & 4.85 & 6.50 \\
\hline 7.35 & 8.01 & 8.27 & 12.06 & 31.75 & 32.52 & 33.91 & 36.71 & 72.89 & \\
\hline
\end{tabular}

Moreover, we can obtain from the sample $(*)$ the following eight upper records:

\begin{tabular}{llllllll}
\hline 0.19 & 0.96 & 4.15 & 8.01 & 31.75 & 33.91 & 36.71 & 72.89 \\
\hline
\end{tabular}

Based on these fourteen PCOs with $\bar{R}^{*}=(0, \ldots, 0,5)$, the results of DFPI of future $r$ th PCOs $X_{r: 10: 15}, 1 \leq r \leq 10$ from unobserved PCOs of size 10 with $\bar{R}=(0, \ldots, 0,5)$ from a future Y-sample of size 15 are displayed in table 7. Also, based 
on the above order statistics obtained, DFPI of future $r$ th PCOs $X_{r: 10: 15}, 1 \leq r \leq 10 \leq 15$ from a future unobserved random sample of size 10 with $\bar{R}=(0, \ldots, 0,5)$ from a future Y-sample of size 15, with corresponding prediction coefficient of at least 0.80 are displayed in table 8 . In table 9 , we have presented the values of $\pi_{3}(1,5 ; r)$ for $m: k=100: 90$, for some selected choices of $r$ based on the real observed upper records. Such $\pi_{3}(i, j ; r)$ is given by (13).

Table 7. PIs for future $r^{\text {th }}$ PCOs $Y_{r: 10: 15}, 1 \leq r \leq 10 \leq 15$ from a future unobserved random sample of size 10 from Y-sample, based on the real observed PCOs.

\begin{tabular}{llllllll}
\hline$r$ & $(i, j)$ & $\left(X_{i: 14: 19}, X_{j: 1419}\right)$ & $\pi_{1}$ & $r$ & $(i, j)$ & $\left(X_{i: 14: 19}, X_{j: 14: 19}\right)$ & $\pi_{1}$ \\
\hline & $(1,5)$ & $(0.19,3.16)$ & 0.5170 & 6 & $(3,13)$ & $(0.96,32.52)$ & 0.7318 \\
& $(1,5)$ & $(0.19,3.16)$ & 0.6251 & 7 & $(5,14)$ & $(3.16,33.91)$ & 0.6423 \\
& $(1,7)$ & $(0.19,4.85)$ & 0.6987 & 8 & $(5,14)$ & $(3.16,33.91)$ & 0.4364 \\
& $(1,9)$ & $(0.19,7.35)$ & 0.7203 & 9 & $(5,14)$ & $(3.16,33.91)$ & 0.2186 \\
$(2,12)$ & $(0.78,31.75)$ & 0.8012 & 10 & $(5,14)$ & $(3.16,33.91)$ & 0.0614 \\
\hline
\end{tabular}

Table 8. DFPI for future $r^{\text {th }}$ PCOs $Y_{r: 10: 15}, 1 \leq r \leq 10$, based on the real observed ordered statistics sample.

\begin{tabular}{llllllll}
\hline$r$ & $(i, j)$ & $\left(X_{i: 19}, X_{j: 19}\right)$ & $\pi_{2}$ & $r$ & $(i, j)$ & $\left(X_{i: 19}, X_{j: 19}\right)$ & $\pi_{2}$ \\
\hline 1 & & & & 6 & $(2,14)$ & $(0.96,8.27)$ & 0.8042 \\
2 & $(1,8)$ & $(0.19,2.78)$ & 0.8045 & 7 & $(5,16)$ & $(2.78,12.06)$ & 0.8246 \\
3 & $(1,9)$ & $(0.19,2.78)$ & 0.8181 & 8 & $(7,18)$ & $(2.78,12.06)$ & 0.8778 \\
4 & $(1,12)$ & $(0.19,2.78)$ & 0.8186 & 9 & $(3,19)$ & $(2.78,12.06)$ & 0.8891 \\
5 & $(3,13)$ & $(0.78,8.01)$ & 0.8295 & 10 & & & \\
\hline
\end{tabular}

Table 9. DFPI for some future $r^{\text {th }}$ PCOs $Y_{r: 90: 100}, 1 \leq r \leq 90 \leq 100$ from a future unobserved random sample of size 90 from Y-sample, based on above upper records.

\begin{tabular}{llllllll}
\hline$r$ & $(i, j)$ & $\left(U_{1}, U_{5}\right)$ & $\pi_{3}$ & $r$ & $(i, j)$ & $\left(U_{1}, U_{5}\right)$ & $\pi_{3}$ \\
\hline 10 & $(1,5)$ & $(0.19,31.75)$ & 0.0990 & 60 & $(1,5)$ & $(0.19,31.75)$ & 0.5913 \\
20 & & 0.1980 & 70 & & 0.6848 \\
30 & & 0.2970 & 80 & & 0.7674 \\
40 & & 0.3958 & 90 & & 0.8085 \\
50 & & & 0.4942 & & & \\
\hline
\end{tabular}

\section{Conclusion}

In this article, based on progressive Type-II right-censored, ordered statistics, record values and current records, distribution-free PIs for future progressively Type-II right censored order statistics are derived. The obtained results in all tables show that the DFPI decreases with decreasing PI length. Also, the corresponding coefficient increases with decreasing the past sample size. Moreover it may be noted from the results, the prediction coefficients for a future $r^{\text {th }}$ PCOs $Y_{r: k: m}, 1 \leq r \leq k \leq m$ increase as $k$ increase, even if the censored observations size $m-k$ increase. Based on results of tables (5,6 and 8), DFPI for PCOs based on upper (current) records may be advised only for higher $r^{\text {th }}$ PCOs.

\section{References}

[1] D. Krewski, Distribution-free confidence intervals for quantile intervals, J. American Stat. Association, 71 (1976) 420 - 422.

[2] J. Ahmadi, N. Balakrishnan, Confidence intervals for quantiles in terms of record range, Stat. Prob. Letters 68 (2004) $395-$ 405 .
[3] J. Ahmadi, N. Balakrishnan, Distribution-free confidence intervals for quantile intervals based on current records, Stat. Prob. Letters 75 (2005) $190-202$.

[4] J. Ahmadi, N. Balakrishnan, outer and inner prediction intervals for order statistics based on current records, Springer, 53 (2012) 789- 802 .

[5] M. Z. Raqab, N. Balakrishnan, Prediction intervals for future records, Stat. Prob. Letters, 78 (2008) $1955-1963$.

[6] J. Ahmadi, N. Balakrishnan, Prediction of order statistics and record values from two independent sequences, Statistics, 44 (2010) 417-430.

[7] J. Ahmadi, N. Balakrishnan, Distribution-free prediction intervals for order statistics based on record coverage, J. Korean Stat. Society, 40 (2011) $181-192$.

[8] J. Ahmadi, S.M.T.K. MirMostafaee, N. Balakrishnan, Nonparametric prediction intervals for future record intervals based on order statistics, Stat. Prob. Letters, 80 (2010) 1663 1672.

[9] M.Z. Raqab, Distribution-free prediction intervals for the future current record statistics, Springer, 50 (2009) 429- 439.

[10] M. Z. Raqab, N. Balakrishnan, Prediction intervals for future records, Stat. Prob. Letters, 78 (2008) $1955-1963$. 
[11] M. M. Mohie El-Din, M. S. Kotb, W. S. Emam, Prediction intervals for future order statistics from two independent sequences, AJTAS, 4 (2015) $33-40$.

[12] U. Kamps, E. Cramer, On distributions of generalized order statistics, Statistics, 35 (2001) $269-280$.

[13] E. Basiri, M. Z. Raqab, Nonparametric prediction intervals for progressive Type-II censored order statistics based on k-records, Springer, 28 (2013) 2825 - 2848.

[14] R. Aggarwala, N. Balakrishnan, Progressive censoring: theory,methods, and applications. Birkhäuser, Boston (2000).

[15] N. Balakrishnan, Progressive censoring methodology: an appraisal, Springer, 16 (2007) $211-259$.

[16] N. Balakrishnan, A. Childs, B. Chandrasekar, An efficient computational method for moments of order statistics under progressive censoring, Stat. Prob. Letters, 60 (2002) 359-365.

[17] B.C. Arnold, N. Balakrishnan, H.N. Nagaraja, A First Course in Order Statistics, John Wiley - Sons, New York, (1992).
[18] V.B. Nevzorov, Records, Mathematical Theory (English Translation), American Mathematical Society, Providence, (2000).

[19] S. Gulati, W.J. Padgett, Parametric and Nonparametric Inference from Record-Breaking Data,Springer-Verlag, New York, (2003).

[20] M. Ahsanullah, Record Values - Theory and Applications, University Press of America Inc., New York, (2004).

[21] B.C. Arnold, N. Balakrishnan, H.N. Nagaraja, Records, John Wiley - Sons, New York, (1998).

[22] W.B. Nelson, Applied life data analysis, Wiley, New York, (1982).

[23] J.F. Lawless, Statistical model \& methods for lifetime data, Wiley, New York, (1982).

[24] H.A. David, H.N. Nagaraja, Order Statistics, third ed. John Wiley - Sons, Hoboken, New Jersey, (2008). 